\title{
Pathogenesis, diagnosis and treatment of non-alcoholic fatty liver disease
}

\author{
Verónica Martín-Domínguez, Rosario González-Casas, Jorge Mendoza-Jiménez-Ridruejo, Luisa García- \\ Buey and Ricardo Moreno-Otero
}

\begin{abstract}
Digestive Diseases Service and CIBEREHD (Instituto de Salud Carlos III). Hospital Universitario de La Princesa. Instituto de Investigación Sanitaria Princesa (IIS-IP). Universidad Autónoma de Madrid. Madrid, Spain
\end{abstract}

\begin{abstract}
Non-alcoholic fatty liver disease (NAFLD) includes a broad spectrum of alterations that go from simple steatosis to steatohepatitis and cirrhosis. Type 2 diabetes mellitus (DM-2) and obesity are the principle factors associated to NAFLD. A $20-30 \%$ prevalence in general population has been described. The survival of this type of patient is lower than the general population's, showing a higher incidence of hepatic and cardiovascular complications. The aetiopathogenesis is still unclear, but we know the intervention of different factors that produce fatty-acid accumulation in hepatic parenchyma, causing oxidative stress, oxygen-free radicals and the synthesis of an inflammatory cascade, that determine the progression of this disease from steatosis up to advanced fibrosis.

The diagnostic gold-standard is still the liver biopsy, even though the development of newer non-invasive techniques, like serological and imaging (radiology), have opened a new field for research that allows bloodless testing of these patients and better study of the natural history of this disease. Nowadays, there is still no specific treatment for NAFLD. The development of healthy life habits and moderate exercise continue to be the pillars of treatment. Different pharmacological approaches have been studied and applied, such as the control of insulin resistance, lowering cholesterol levels, antioxidants, and other alternatives in experimental trials.
\end{abstract}

Key words: Steatosis. Steatohepatitis. Fibrosis. Non-alcoholic fatty liver disease (NAFLD).

Martín-Domínguez V, González-Casas R, Mendoza-JiménezRidruejo J, García-Buey L, Moreno-Otero R. Pathogenesis, diagnosis and treatment of non-alcoholic fatty liver disease. Rev Esp Enferm Dig 2013;105:409-420.

Received: 21-09-2012

Accepted: 03-04-2013

Correspondence: Ricardo Moreno-Otero. Department of Digestive Diseases (3rd floor). Hospital Universitario de La Princesa. C/ Diego de León, 62. 28006 Madrid, Spain

e-mail: rmorenoo@salud.madrid.org

\section{ABBREVIATIONS}

ALT: Alanine aminotransferase.

ARFI: Acoustic radiation force impulse.

AST: Aspartate aminotransferase.

BMI: Body mass index.

CAP: Controlled attenuation parameter.

CRP: C-reactive protein.

CT: Computed tomography.

DM-2: Diabetes mellitus type 2.

FFA: Free fatty acids.

FLD: Fatty liver disease.

FLI: Fatty liver index.

FXR: Farnesoid X receptor.

GGT: Gamma glutamyl transpeptidase.

GIP: Gastric inhibitory peptide.

GLP-1: Glucagon-like peptide-1.

HCC: Hepatocellular carcinoma.

HNE: 4-hydroxynonenal.

HSL: Hormone-sensitive lipase.

IL: Interleukin.

LDL: Low-density lipoprotein.

LPS: Lipopolysaccharide.

M-CSF: Macrophage colony stimulating factor.

MDA: Malondialdehyde.

MRC: Mitochondrial respiratory chain.

MRI: Magnetic resonance imaging.

MRS: Magnetic resonance spectroscopy.

NASH: Non- alcoholic steatohepatitis.

NFS: NAFLD fibrosis score.

OFR: Oxygen free radicals.

PPAR: Peroxisome proliferative activated receptor.

PTX-3: Plasma pentraxin 3.

TGF- $\beta 1$ : Transforming growth factor beta.

TNF $\alpha$ : Tumour necrosis factor alpha.

UDCA: Ursodeoxycholic acid.

VEGF: Vascular endothelial growth factor.

VLDL: Very low-density lipoprotein. 


\section{INTRODUCTION}

The non-alcoholic fatty liver disease (NAFLD) can develop a wide variety of clinical and pathological manifestations that are undistinguishable from those observed in alcoholic patients (1). The NAFLD is secondary to the fat accumulation, especially in the form of triglycerides in the hepatocytes and these patients may show simple hepatic steatosis lesions, steatosis with inflammation (non-alcoholic steatohepatitis, NASH), cirrhosis and hepatocellular carcinoma (HCC) (2).

Diabetes mellitus type 2 (DM-2) and obesity are the principal factors associated with NAFLD, which is considered the hepatic manifestation of the metabolic syndrome, and its prevalence increases in parallel with these two diseases. It is estimated that NAFLD is the first cause of chronic elevation of liver function texts in the USA and it is believed that cryptogenetic cirrhosis may be associated with it (3).

\section{EPIDEMIOLOGY}

The prevalence of NAFLD in western countries is 20-30 \% and about $15 \%$ in Asian countries. It has been observed in all age groups, including children, where the prevalence is $10-15 \%$ (less than in adults). As a general rule, the prevalence increases with age, affecting equally both sexes (3). Between $90-100 \%$ of the patients diagnosed with obesity present some degree of NAFLD. Amongst those who have DM-2, the prevalence is around 10-75\% and in those with hyperlipidaemia it is between 20-35\%, and in patients with cirrhosis around $3 \%(1,4)$. NAFLD may be the most frequent cause of persistent hypertransaminasemia and cryptogenetic cirrhosis in adults (5).

The variability of manifestations, of progression and the natural course of this disease amongst individuals of different race, and the incidence observed in some families, has awakened the interest in the studies of genetic mutations that could determine a genetic predisposition. For now the genes responsible for this remain unknown, but several candidates have been proposed: a) genes related to abdominal obesity and DM-2; b) genes that suppress the exit of very low-density lipoprotein (VLDL) from the hepatocytes; c) mutations and polymorphism of genes associated with oxidative stress or of the protecting enzyme superoxide dismutase; and d) genes responsible of inflammatory response and fibrogenesis (6). Among these mutations under study we find the over-expression of genes like TNF- $\alpha$, M-CSF (macrophage colony stimulating factor) and TRAIL-R2 (apoptosis mediated receptor) (7). The existence of a nonsynonymous polymorphism for rs738409 in the gene PNPLA3 (8) predicts the future outcome of patients with NAFLD and its association with fibrosis. Today's studies are focussed on "genome-side association" type analysis in order to identify markers for the severity of NAFLD (6).

\section{NATURAL HISTORY}

The progression of this liver condition depends on the level of histological damage at the time of diagnosis, meaning that fatty liver disease (FLD) tends to remain more or less stable while non-alcoholic fatty liver disease (NAFLD) tends to lead to cirrhosis and hepatocellular carcinoma (HCC). The gold standard diagnosis is liver biopsy, but with this long-duration liver disease it is difficult to follow large groups of people by means of serial biopsies; in an attempt to avoid this invasive method the diagnostic and prognostic implementation of different non-invasive methods has been studied (9).

In a developmental study of 132 patients during a 10 -year follow-up period it was revealed that $25 \%$ of patients with NAFLD had progressed to cirrhosis $-12 \%$ died due to liver disease (7). The factors that imply a greater risk of progression of NAFLD are: Age over 40 years, body mass index $(\mathrm{BMI})>40 \mathrm{~kg} / \mathrm{m}^{2}$, an AST (aspartate aminotransferase)/ALT (alanine aminotransferase) ratio greater than 1 and the coexistence of DM-2 or hyperlipidaemia. Mortality associated with NAFLD depends on the stage of the disease, since patients with FLD or minimal fibrosis have a very low risk of death after 10 years. However, those with more advanced lesions are at risk of suffering complications such as hepatocellular failure, bleeding varices, ascites, hepatorenal syndrome and HCC. The risk of mortality is higher than in patients with cirrhosis from other causes, due to the association of NAFLD with metabolic factors and cardiovascular risk $(3,10)$. A histological improvement in some patients with NAFLD has also been described (9). After losing weight, a decrease in inflammation and even perisinusoidal fibrosis is detected, especially if weight loss is gradual and diet is associated with physical exercise (11). In some cases, liver failure occurs due to rapid weight loss, regardless of the cause, but especially in morbidly obese patients undergoing bariatric surgery $(3,10)$.

\section{PROGNOSIS}

NAFLD is described in close relation to the universal obesity epidemic, that is to say, the process has a metabolic basis and its severity varies. FLD has a good prognosis, but patients who do not correct their metabolic problem tend to have an unfavourable evolution. By contrast, patients with NAFLD may progress to cirrhosis and develop HCC. The age and the presence of inflammation in the initial biopsy were independent predictors of NAFLD lesion progression to advanced fibrosis and cirrhosis (12).

In patients with cirrhosis secondary to NAFLD, HCC and hepatocellular failure are leading causes of morbid-mortality. The cumulative rate of development of HCC was $11.3 \%$, with a survival rate after 5 years of $75.2 \%$. The cumulative proportion of $\mathrm{HCC}$ recurrence after a 5-year 
follow-up period was $72.5 \%$. The conclusions drawn from these data are of paramount importance and show the need for protocol monitoring of patients with NAFLD for the prevention of cirrhosis complications, with endoscopy in order to evaluate the formation of oesophageal varices and with ultrasound for early diagnosis of HCC (12).

NAFLD has an increased risk of cardiovascular disease-related death and may indicate future cardiovascular events, independently of sex, age, LDL (low-density lipoprotein)-cholesterol levels and tobacco consumption. There are multiple studies linking elevated liver enzyme levels (mainly gamma-GT) with high risk of stroke or heart disease. Hoorn's study of a cohort of Caucasian population between 50-75 years found an increased risk of heart disease or cardiovascular disease or related to ALT elevations over baseline (13). A recent study showed that patients with NAFLD have a significantly increased risk of cardiovascular and kidney disease (14), even in patients transplanted for NAFLD. There is an abundance of basic and prospective clinical studies on the activity of pentraxin 3 (PTX3) in relation to the inflammatory processes associated with cardiovascular diseases. Its primary expression is arteriosclerotic lesions and in-depth investigation is underway to see if plasma concentrations are valid for use as a new biomarker of cardiovascular inflammation (15). In regard to patients with NAFLD, there are no results at present although an abundance of research in this line of study exists.

\section{PATHOGENESIS}

The numerous clinical situations that have been associated with NAFLD can be grouped into 6 etiological groups (3) (Table I). However, there is a high association -up to $80 \%$ - between this liver disease and the metabolic syndrome. The histological signs of FLD are especially characteristic in the obese population and in patients with DM-2 (16). These two conditions have been associated with peripheral insulin resistance and glucose intolerance. Dyslipidaemia, particularly hypertriglyceridaemia, is also associated with steatosis. However, despite the fact that the majority of patients are overweight and obese, NAFLD may also occur in patients with normal weight and may constitute an independent cardiovascular risk factor (10).

The pathogenic mechanism of NAFLD is associated with insulin resistance and can be explained as the 'double impact theory $(11,17)$. In the "first impact", the reduction in cellular capacity to respond to the action of insulin causes compensatory hyperinsulinaemia. In adipose tissue it acts on the hormone-sensitive lipase (HSL) increasing the risk of lipolysis with the consequent release of free fatty acids (FFA) to the liver. Glucose absorption decreases in the skeletal muscle, while in the hepatocyte hyperinsulinaemia increases gluconeogenesis, decreases glycogen synthesis and increases uptake of FFA, alters the transport
Table I. Classification of different causes associated with NAFLD

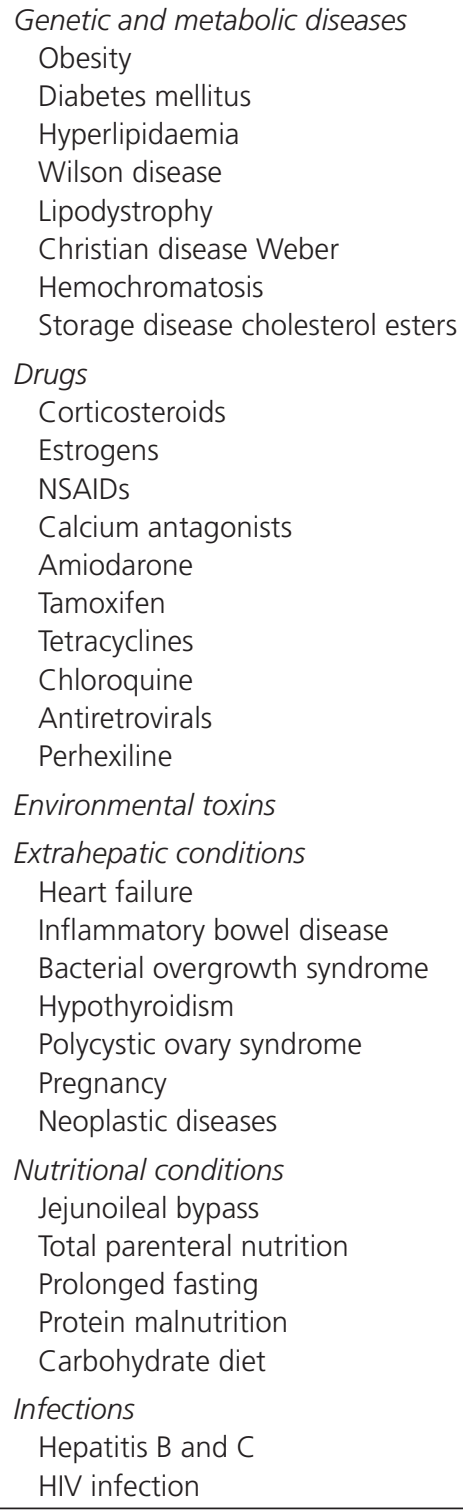

of triglycerides such as VLDL and inhibits beta-oxidation. These alterations in the metabolism of fats are the basis of FLD (11) (Fig. 1). This "first impact" results from the interaction of various factors, such as hepatic resistance to leptin or the reduction of adiponectin levels, it would therefore be more correct to speak of "multiple impacts", with a predominance of one or the other, depending on the patient.

The "second impact" is a consequence of oxidative stress in hepatocytes, which is initially compensated by cellular antioxidant mechanisms. However, liver overload of FFA generates oxygen free radicals (OFR) in the mitochondrial chain that act upon the fatty acids of the 
cell membranes causing lipid peroxidation. OFR induce proinflammatory cytokine synthesis due to Kupffer cells and hepatocytes, such as: a) tumour necrosis factor-alpha (TNF- $\alpha$ ), which activates the caspase pathway and leads to hepatocyte apoptosis; b) transforming growth factor beta-1 (TGF- $\beta 1$ ), which activates collagen synthesis due to stellate cells; c) Fas ligand that cause 'fratricide deaths' between adjacent hepatocytes; and d) interleukin-8 (IL-8), powerful neutrophil chemotactic. The end products of lipid peroxidation, 4-hydroxynonenal (HNE) and malondialdehyde (MDA), are also involved in the pathogenesis of liver damage due to direct toxicity, and can intervene in the formation of Mallory body and increase collagen synthesis due to stellate cells; HNE also has neutrophil chemotactic activity (18-22). This second phase would explain the evolution necroinflammatory phenomenon, fibrosis and liver cirrhosis (Fig. 1). Various studies (23-28) outline other factors involved in the pathogenesis of NAFLD:

1. Insulin resistance causes elevated serum ferritin levels, increased expression of transferrin receptors and increased hepatic iron, which contributes to the generation of hydroxyl radicals and the accumulation of oxygen free radicals (OFR), however, its role in the pathogenesis of NAFLD is yet to be clarified (23).

2. It also involves the generation of adipokines due to adipose tissue. Due to its endocrine, paracrine and systemic effects, they behave like hormones and may play a proinflammatory role in NAFLD.

3. The role of adiponectin is widely reported due to its anti-inflammatory, antiatherosclerotic, anti-lipogenic and hypoglycaemic effects, and also due to its protective mechanism of fatty liver development and its ability to act on stellate cells inhibiting fibrogenesis (23). Two adiponectin receptors have been identified: type I - in the skeletal muscle and type II - in the liver, whose decrease has been associated with a greater degree of steatosis in patients with similar adiponectin levels, although there was increased expression of its receptors with NAFLD progression (24). Obesity (especially visceral) and being overweight have

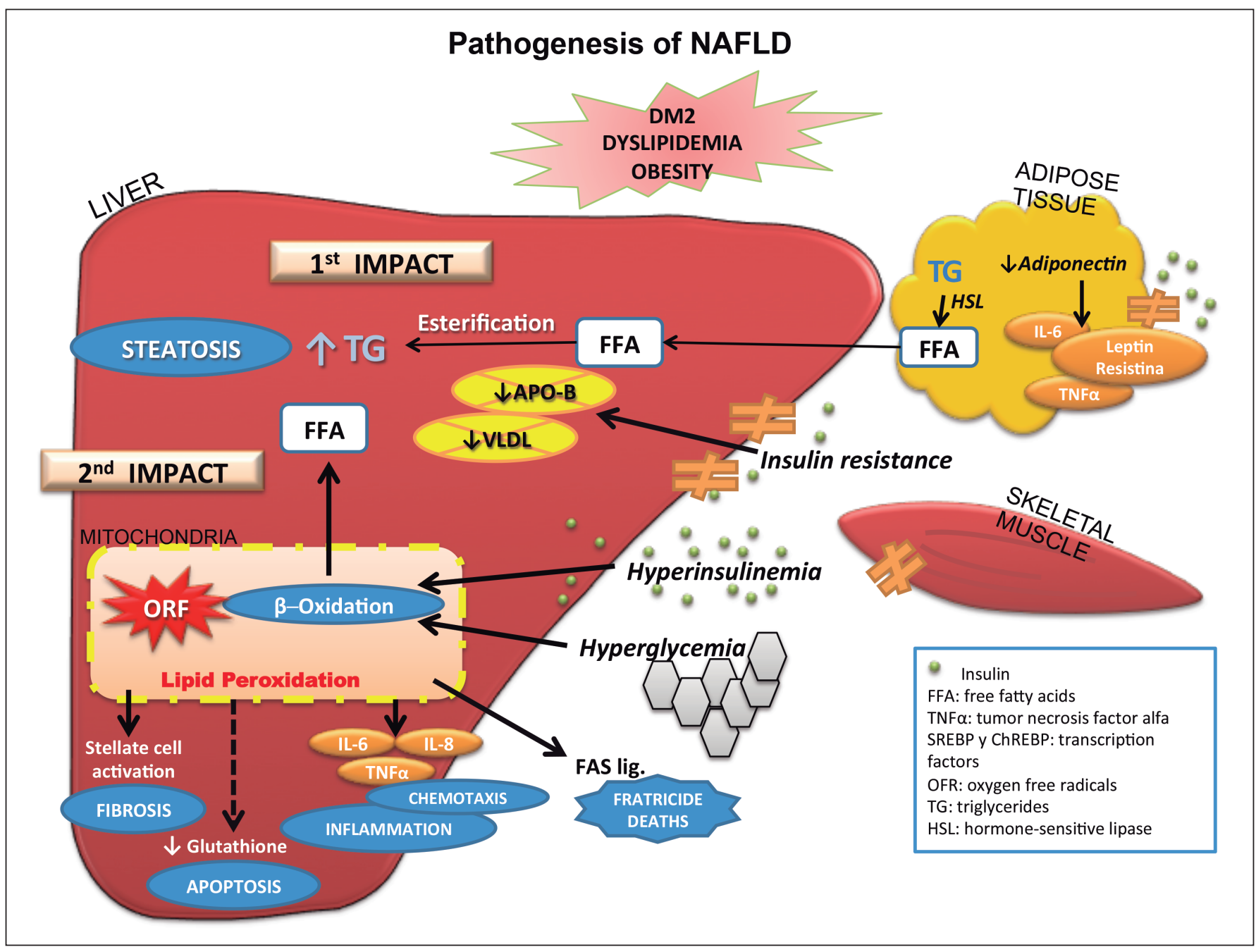

Fig. 1. Pathogenesis of NAFLD (author: Verónica Martín). 
been associated with low adiponectin levels due to TNF- $\alpha$ inhibition - this imbalance may be one of the pathophysiological mechanisms of NAFLD and the regulation of pathways that control production and signalling may represent a promising therapeutic target (25). The decrease in adiponectin may be the "expression in the organ of metabolic syndrome".

4. Leptin is another cytokine whose primary resistance was described at hypothalamic level in the nuclei of satiety control, although, at present, insulin resistance is associated with peripheral leptin resistance in the skeletal muscle. In patients with NAFLD, serum leptin concentration levels are elevated and are related to the degree of steatosis (26). However, they are not correlated with the degree of fibrosis and do not appear to improve or reverse the problem - the "leptin resistance theory" has thus been proposed, which is associated with obesity, insulin resistance and elevated glucose levels in patients with NAFLD (26).

5. Bacterial overgrowth and increased bacterial translocation to portal and systemic circulation, as well as increased serum levels of lipopolysaccharide (LPS), bacteria and endotoxin and the activation of proinflammatory signalling (TNF- $\alpha$ and IL-6) have been observed in various chronic liver diseases including NAFLD (27).

6. The cannabinoid system plays a fundamental role in the pathophysiology of chronic liver disease. The positive regulation of the endocannabinoid system during chronic liver disease participates in the pathogenesis of hepatocyte necrosis, inflammation and fibrogenesis (28).

\section{DIAGNOSIS}

Established on the basis of various clinical application tools (Table II) that are exposed as follows:

\section{Clinical evaluation}

- Physical and anthropometric examination: The majority of patients with NAFLD are asymptomatic at diagnosis (50-75 \%) or present non-specific symptoms such as asthenia, general malaise and pain in the right upper quadrant (30-40\%). Only 5-10\% present with decompensated liver disease. On physical examination, hepatomegaly (55-85\%) and signs of advanced liver disease are common.

- Exclusion of excessive alcohol consumption: A basic diagnostic criteria for what should be rigorously excluded through a thorough and careful examination of the patient and often of family members and friends. Diagnosis of NAFLD cannot be established in patients with alcoholic ingestion $>20 \mathrm{~g}$ of alcohol/

\section{Table II. Diagnostic evaluation of NAFLD}

Clinical evaluation
Physical examination and anthropometric
Exclusion of excessive alcohol consumption
Exclusion of other causes of fatty liver nonalcoholic aetiology
Serum markers and biochemical indices
Biochemical tests
Biomarkers and direct markers of inflammation and fibrosis
Mathematical models that include clinical and laboratory
variables
Imaging tests
Ultrasonography
Computed tomography (CT)
Nuclear magnetic resonance (NMR)
Transition elastography (FibroScan ${ }^{\circledR}$ )
New imaging techniques
Liver biopsy

day in women and $>30 \mathrm{~g} /$ day in men. It is sometimes important to rely on analytical parameters that may indicate active alcoholism (5). Currently, the parameter of higher sensitivity and specificity as a marker of chronic alcoholism is the desialylated transferrin/ total transferrin ratio $>1$ (27). In cases of poor communication with the patient or family, determination of alcoholaemia or alcoholuria is required.

- Exclusion of other causes of chronic liver disease: All the necessary studies must be carried out (biochemical and immune-serological) in order to exclude other aetiologies (viral, autoimmune, metabolic or toxic).

\section{Serum markers and biochemical indices}

- Biochemical tests: Up to $80 \%$ of patients with NAFLD have normal liver function tests. The elevation of transaminases is rare and usually less than 4 times the normal value, rarely surpassing 10-15 times. However, the levels do not correlate with the degree of FLD or fibrosis. Unlike alcoholic liver disease, the ALT/AST $>1$ ratio is present in $85-90 \%$ of cases. Elevated gamma glutamyl transpeptidasa (GGT) and ALP (double or triple) in $50 \%$ of patients is also frequent. Bilirubin and albumin usually remain normal until advanced stages of liver disease (29). 20-50\% of these patients have elevated ferritin levels and increased transferrin saturation. However, none of these data are specific for the diagnosis of NAFLD and provide no information on the aetiology and do not differ between stages of FLD and NAFLD (29).

- Biomarkers and direct markers of inflammation and fibrosis: Several non-invasive methods are being studied to assess the presence of steatosis, necroinflammation and fibrosis, in order to avoid liver biopsy. It is 
important to identify the degree of inflammation, as it plays a central role in the progression of NAFLD (29). C-reactive protein (CRP) is an acute-phase reactant synthesized in the liver, whose plasma levels may be useful in differentiating between FLD and NAFLD. Plasma pentraxin 3 (PTX3) is a new acute-phase reactant that permits assessment of the fibrosis stage, in correlation with IL-6 levels, and is also a cardiovascular marker of inflammation (15). TNF- $\alpha$ is highly expressed in NAFLD and cytokeratin-18 is a relatively new marker of apoptosis and the activity and severity index of NAFLD $(4,29)$. Although several parameters that determine oxidative stress (glutathione peroxidase activity, superoxide dismutase activity and vitamin E levels) have been evaluated, their clinical usefulness has not been demonstrated $(18,22)$.

- Mathematical models that include clinical and laboratory variables: SteatoTest (BioPredictive), Fibrospect and "NAFLD fibrosis score" (NFS). The SteatoTest is a qualitative analysis that estimates FLD, evaluating nine biochemical markers (alpha 2-macroglobulin, haptoglobin, apolipoprotein 1, total bilirubin, gamma-GT, fasting glucose, triglycerides, cholesterol, and ALT) adjusted for the patient's age, sex, weight and height. This test has been validated for the assessment of NAFLD, with $33 \%$ sensitivity and $94 \%$ specificity (30). Fibrospect evaluates fibrosis by means of analysis of markers such as hyaluronic acid, a metalloproteinase inhibitor, $\alpha-2$ macroglobulin, gamma-GT, haptoglobin, bilirubin and apolipoprotein $(29,30)$. NFS uses 6: Age, body mass index (BMI), diabetes, AST/ALT ratio, platelets and albumin; evaluated in a cohort of 733 patients with NAFLD and liver biopsy, their cut-off points permit the presence or absence of advanced fibrosis (30) to be predicted. Finally, fatty liver index (FLI) values, BMI, waist circumference, triglycerides and gamma-GT - 1,300 apparently healthy individuals studied showed that it predicts insulin resistance and cardiovascular risk factors, apart from having potential value for detecting NAFLD in the general population (29).

\section{Imaging tests}

- Ultrasonography, computed tomography (CT) and magnetic resonance imaging (MRI) (29-31): Abdominal ultrasound is the first technique of choice for the diagnosis of NAFLD. It evaluates the presence of steatosis, with sensitivity between $60 \%$ and $94 \%$, although it is very low when the degree of steatosis is below $30 \%$, when specificity is close to $90 \%$ (29). According to these authors, the longitudinal diameter of the spleen may differentiate between FLD and NAFLD (when the value is greater than $116 \mathrm{~mm}$ ) (29), and can be more effective than the determination of
IL-6 or vascular endothelial growth factor (VEGF). In the protocol of the Canadian Association of Radiology (31), the following findings are positively correlated with NAFLD: a) Increased echogenicity in relation to the kidney; b) non-affected areas, defined as specific areas of the liver without fatty infiltration; c) visualization of portal vessels and diaphragm; d) sound attenuation; and e) hepatomegaly.

CT is applicable in order to assess thickening of subcutaneous abdominal adipose tissue and to measure liver fat. It detects splenomegaly secondary to portal hypertension, suggesting advanced fibrosis due to NAFLD. CT permits classification of FLD by calculating the relation between the attenuation of the liver and spleen. Its limitations are the difficulty in identifying the intermediate stages of fibrosis and its application in monitoring programs due to radiation (29).

MRI offers a quick and accurate assessment of FLD, even at a level below $3 \%$. Phase-contrast imaging permits quantitative assessment of fatty infiltration with good histological correlation, permitting differentiation of FLD and NAFLD by means of contrast and $88 \%$ sensitivity (29-31).

- New imaging techniques:

- Transient elastography (FibroScan $\left.{ }^{\circledR}\right)$ : Is a non-invasive method that assesses liver fibrosis and appears to be more sensitive than serological markers (29). The accuracy for evaluating advanced fibrosis and cirrhosis is good, but unreliable in obese patients $(\mathrm{BMI}>28)$, in the presence of ascites and when intercostal space is narrow (30-32). A new parameter called "Controlled Attenuation Parameter" (CAP) has recently been included, which permits efficient assessment of steatosis and fibrosis by means of FibroScan (33).

- Proton magnetic resonance spectroscopy (MRS): Measures the fraction of protons in fat and liver triglyceride levels (HTG). HTG $>5 \%$ is the level for FLD diagnosis. MRS characterizes the metabolic processes involved in cell regeneration, permitting the severity of steatosis in NAFLD to be quantitatively assessed. An increase in ATP/phosphate ratio could be a sign of progression to advanced stages of fibrosis. MRS seems more specific than the above procedures; however validation by means of controlled studies is required (29).

- Acoustic radiation force impulse (ARFI) imaging or elastosonography: Proposed as an alternative method to FibroScan, it uses sound waves to determine the mechanical stiffness of the liver. One advantage of ARFI is that it is integrated into a conventional ultrasound system, and therefore can be performed during standard tests. Preliminary data indicate that ARFI is effective to evaluate hepatic fibrosis, although its effectiveness for the assessment of NAFLD has not yet been established (32). 
- Magnetic resonance elastography (MRE): has higher diagnostic accuracy of fibrosis and is not as conditioned by BMI (29).

\section{Liver biopsy}

This is the gold standard test for the evaluation of NAFLD. It classifies patients according to the severity of steatosis, degree of inflammation and stage of fibrosis, and forecasts its evolution towards cirrosis (4). Histological diagnosis is made by means of the Brunt semi-quantitative classification (34), classifying inflammatory activity in grades from 0-3 and fibrosis stages 0-4 (Table III). The NASH Clinical Research Network recently developed a new scoring system for the quantitative classification of NAFLD, which includes the following criteria: Steatosis, lobular inflammation, hepatocellular ballooning, Mallory bodies and fibrosis. It can differentiate between FLD and NAFLD and assesses fibrosis in five stages; 0 : no fibrosis, 1 : perisinusoidal or portal fibrosis, 2: perisinusoidal and portal/periportal fibrosis, 3: septal or bridging fibrosis, and 4: cirrhosis (35).

A drawback of liver biopsy is the fact that it is an invasive method, which is subject to complications and inter-and intraobserver errors. However, today it is still used in the majority of centres, as, despite technological advances, there is no other method that establishes a complete correlation between clinical data, analytical data and biopsy (4).

\section{TREATMENT}

To date, there is no standardised treatment of NAFLD (Table IV) and no consensus on which is the most appro-

Table III. Modified classification according Brunt

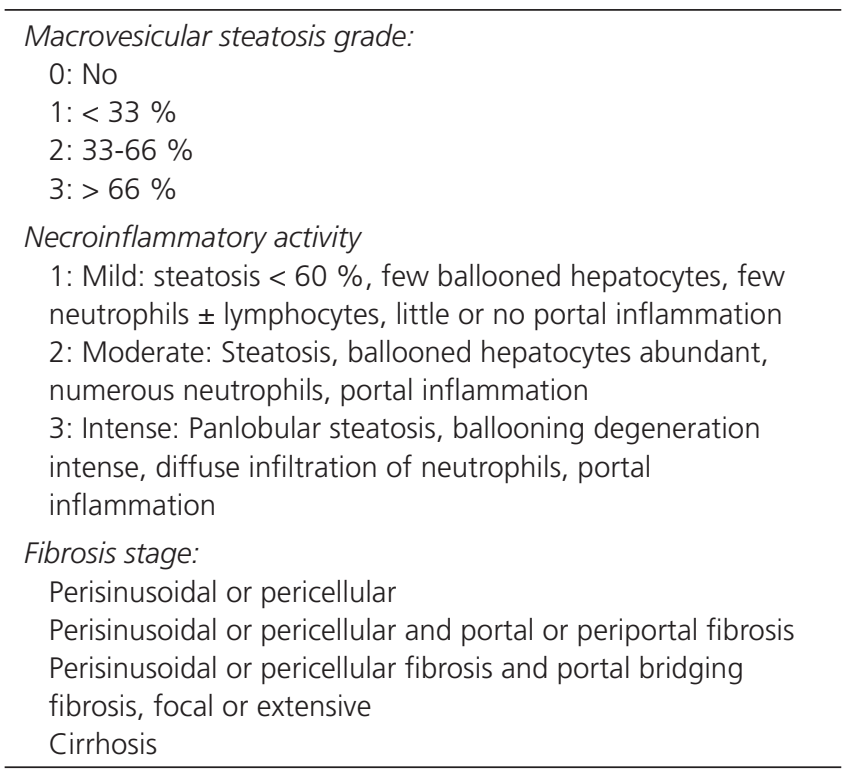

Table IV. Pharmacological and non-pharmacological treatment of NAFLD

\begin{tabular}{l}
\hline No drug therapy \\
\hline Changes in lifestyle: Diet and exercise \\
Avoid sedentary \\
Balanced diet rich in vegetable fiber \\
Exercising continued \\
Avoid sudden weight loss (such as bariatric surgery) \\
Bariatric surgery \\
\hline Drug treatment \\
\hline Treatment of insulin resistance \\
Thiazolidinediones \\
Metformin \\
Incretin mimetics \\
Drugs that induce weight loss \\
Orlistat \\
Sibutramine \\
Cannabinoid receptor blockers: Rimonabant \\
Lipid: Statins \\
Cytoprotective agents and antioxidants \\
Pentoxifylline \\
Ursodeoxycholic acid \\
Tocopherol: Vitamin E \\
N-Acetylcysteine \\
Betaine \\
Blockers angiotensin receptor 2 (ARA II) \\
Other therapeutic options in research \\
Antibiotics and probiotics \\
Receptor agonists farnesoid X \\
Phlebotomies and iron chelation therapy \\
Omega 3 supplements \\
\hline Liver transplantation \\
\hline
\end{tabular}

priate for each patient and the moment to start it. However, the variety of therapeutic guidelines and modes of action are not mutually exclusive options. Firstly, alcohol consumption should be avoided, as well as treatment with potentially hepatotoxic drugs.

\section{Non-pharmacological therapy}

Changes in lifestyle through diet and exercise: comprise the main therapeutic guidelines. Weight loss through caloric restriction, may itself improve transaminase levels, insulin resistance and, in some cases, data from the biopsy (steatosis and lobular inflammation, especially in patients with minimal fibrosis). The best way to lose weight consistently implies a change in eating behaviour, reducing caloric intake to $500-1,000 \mathrm{kcal} / \mathrm{day}$. The goal is a 5-10\% weight loss for 6 to 12 months, as a more rapid loss or stricter diet mobilises fatty liver acids and may lead to increased portal inflammation (36).

Median intensity exercise, like walking 30-45 min/day at a steady pace, improves insulin sensitivity and glucose 
homeostasis (36), reduces VLDL apolipoprotein-B secretion and prevents steatosis, probably as a result of stimulation of lipid oxidation and inhibition of lipid synthesis in the liver through the activation of AMPK (37). These benefits of exercise are independent of weight loss. Liver enzymes are significantly reduced in obese patients with NAFLD by combining sustained weight loss, obtained by means of a hypocaloric diet with increased physical activity (37).

Bariatric Surgery: One of the most effective options to achieve long-term weight loss in severely obese patients. It is indicated in patients with BMI $>35 \mathrm{~kg} / \mathrm{m}^{2}$ with associated comorbidity or BMI $>40 \mathrm{~kg} / \mathrm{m}^{2}$. Cases of severe steatosis caused by rapid weight loss were initially described, especially with jejunoileal bypass. However, proximal gastric-bypass has alleviated this problem. Bariatric surgery in patients with NAFLD has shown the beneficial effects of weight loss on liver histology and liver enzyme elevation (37-39).

\section{Pharmacological therapy (Fig. 2)}

\section{Treatment of insulin resistance}

Thiazolidinediones: Act on the adipose tissue (peroxisome proliferative activated receptor, PPAR- $\gamma$ ), where they increase lipogenesis and sequester fatty acids. This receptor is also a marker of adipocyte differentiation and an essential transcription factor for the expression of adiponectin. The receptor is activated by these drugs and produces an enhancing effect on insulin, facilitates the absorption of fatty acids in adipocytes and their accumulation in the form of triglycerides, and therefore reduces the supply of FFA to the liver (37).

In a systematic review of the value of insulin sensitizers in patients with NAFLD showed that pioglitazone improves all parameters of liver histology (40). However, after the discontinuation of treatment, transaminase levels may return to baseline values (41). The efficacy of rosiglitazone on NAFLD was observed by means of FLIRT study, which revealed a significant antiestrogenic effect in the first year, although there was no additional benefit with longer-term treatment (42). The commercialisation of rosiglitazone was suspended because it caused increased risk of cardiovascular disease.

Metformin: Is a biguanide insulin sensitizer which is widely used in the treatment of DM-2. Its target is the mitochondria where it stimulates beta-oxidation and pyruvate kinase activity, suppressing the expression of lipogenic enzymes. Its main effects are the reduction of hepatic glucose synthesis, the increase of insulin uptake

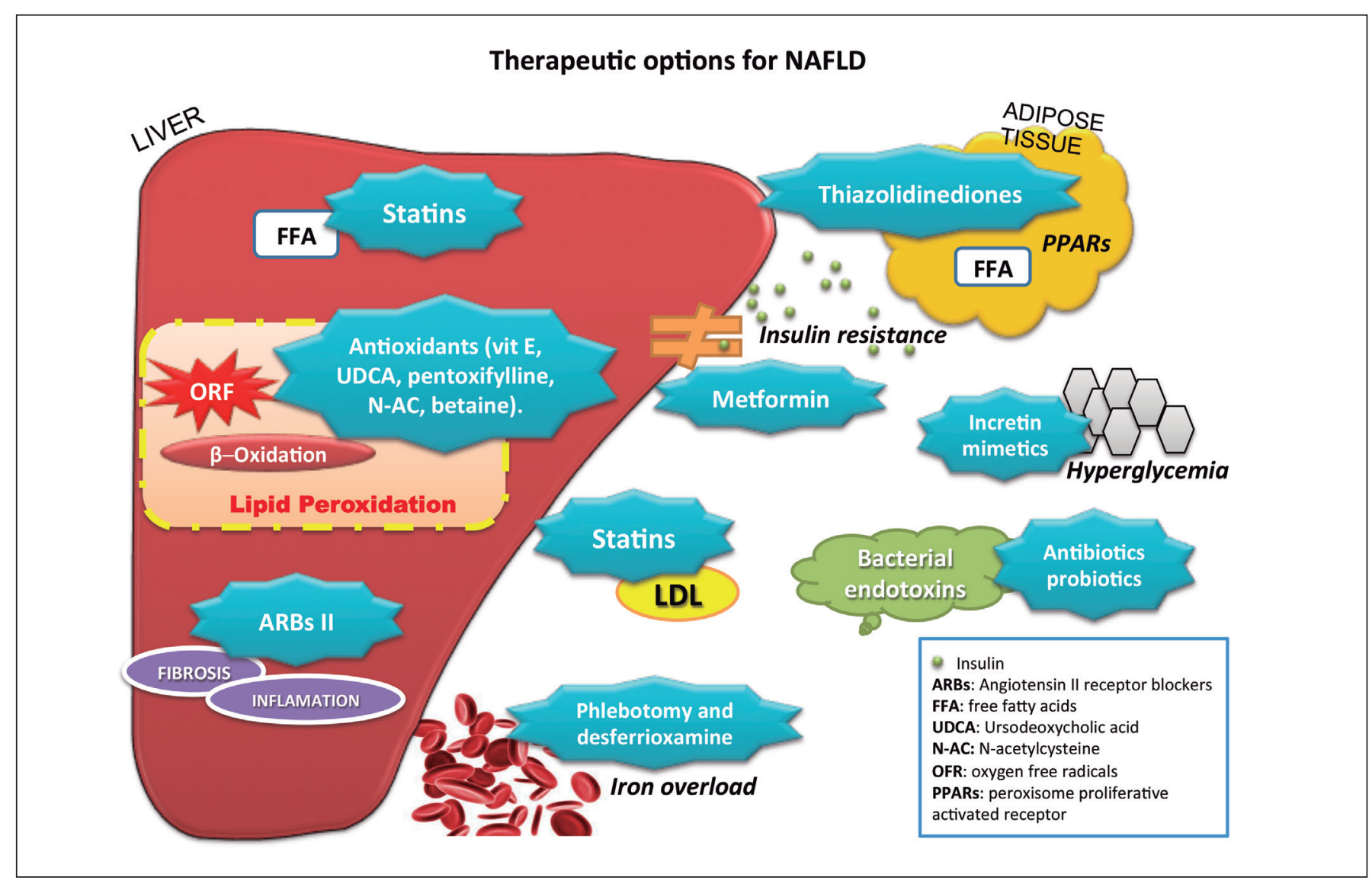

Fig. 2. Mechanisms of action of drug-therapy for NAFLD (author: Verónica Martín). 
in the skeletal muscle and adipocytes, and the reduction of FFA oxidation (43). Transaminase levels and histological damage are reduced in patients with NAFLD treated with metformin (44). A meta-analysis demonstrated that metformin produces a certain improvement in FLD and NAFLD. According to the majority of studies, metformin leads to a significant loss in insulin resistance and weight reduction in patients with NAFLD (40). However, at the present time, guidelines published on NAFLD by AASLD do not recommend its use (45).

Incretin mimetics: Gastric inhibitory peptide (GIP) and glucagon-like peptide-1 (GLP-1): Incretins are proteins that secrete in the intestine when food is ingested and act by stimulating the production of insulin from the beta cells of the pancreas. They have different extra-pancreatic effects including the regulation of hepatic glucose production, the inhibition of pancreatic exocrine secretion, cardioprotective and cardiotropic effects, appetite and satiety regulation, and the stimulation of afferent sensory nerves. Its use is currently reserved for the treatment of diabetes, although it may be a therapeutic option for NAFLD (37).

\section{Drugs that induce weight loss}

Orlistat: produces moderate weight loss by reducing the absorption of fat by $30 \%$ by inhibiting gastric and pancreatic lipases (37). There are studies with mixed results in patients with NAFLD, where there has been no weight loss, improvement in transaminase values or histopathology in overweight patients, compared with the ingestion of a hypocaloric diet and vitamin E supplements. As orlistat causes intestinal malabsorption syndrome, the administration of supplementary vitamins is recommended (46).

Sibutramine: Is a serotonin-norepinephrine reuptake inhibitor of the central nervous system that increases satiety and induces weight loss. It is associated with a decrease in transaminases with a slight increase of alkaline phosphatase and with a reduction of FLD determined by ultrasound. Its long-term effects remain unknown (37).

\section{Cannabinoid receptor blockers: Rimonabant}

Rimonabant is a cannabinoid receptor type 1 antagonist, localised in the liver, adipocytes, skeletal muscle and pancreas. Endocannabinoids are involved in the pathogenesis of NAFLD and its inactivation may reduce apoptosis in patients with fibrosis. It has been shown that one year of treatment with rimonabant significantly reduces liver enzymes in overweight or obese patients with DM-2 compared to placebo. However, it has now been removed from the market because of adverse effects, such as increased depression and suicidal behaviour in some patients, and therefore clinical trials on blockers of endocannabinoids in NAFLD were interrupted (37).

\section{Lipid-lowering drugs: Statins}

Statins cause competitive inhibition of HMG-CoA reductase which leads to a decrease in LDL in the blood. They have pleiotropic effects due to their anti-inflammatory, immunomodulatory and anti-oxidant properties, as well as endothelial dysfunction and the bioavailability of nitric oxide. Major side effects are hepatotoxicity and myopathy, which depend on an idiosyncratic mechanism (47). In the majority of patients with NAFLD there are several components of the metabolic syndrome. Cardiovascular disease remains the leading cause of death, and the reason why statin therapy is indicated. Therapeutic effects include a decrease of transaminases and of steatosis, determined by ultrasound, and histological improvement of liver inflammation (37).

A recent study failed to demonstrate any beneficial effect of simvastatin treatment on biochemical and histological parameters in obese patients with NAFLD (48). On the contrary it showed a significant decrease in transaminases with atorvastatin treatment. The efficacy of rosuvastatin was also observed in patients with hyperlipidaemia, as well as fatty liver and cardiovascular risk reduction (50). It remains to be shown whether liver fibrosis is favourably affected by treatment with statins (51).

\section{Antioxidants and cytoprotective agents}

Oxidative stress is involved in the pathogenesis of NAFLD, therefore antioxidants may reduce liver damage caused by free radicals (52).

- Pentoxifylline inhibits TNF- $\alpha$ synthesis and therefore seems to have a favourable effect on liver enzyme activity, inhibiting lipid oxidation more than placebo (53). Its effect is dose dependent and for prolonged periods, reversing when treatment is stopped.

- Ursodeoxycholic acid (UDCA) is a hydrophilic bile acid with membrane-stabilising properties and cytoprotective and immunomodulatory agents, which appears to decrease AST and steatosis by ultrasound, with high doses $(15-20 \mathrm{mg} / \mathrm{kg} /$ day $)$ maintained for extended periods of time (2 years). However, the results are contradictory and randomised controlled studies that prove histological improvement in patients with NAFLD are required (54).

- Tocopherol: Vitamin E inhibits the intrahepatic expression of TGF- $\beta$, the activation of stellate cells, lipid peroxidation and superoxide anion formation and suppresses proinflammatory cytokines such as TNF- $\alpha$. Several studies have demonstrated the efficacy of vitamin $\mathrm{E}$ at varying doses (300-1200 IU/day for at least 6 months) in the normalisation of transaminase levels, but a clear benefit of lobular inflammation or hepatic fibrosis has not been appreciated (37). Vitamin E increases efficacy in combination with thiazolidinediones, metformin or UDCA. A study carried 
out on obese mice showed that tocopherol could protect against NAFLD by reducing liver damage, lipid peroxidation and inflammation, without modifying body mass or hepatic steatosis. The mechanisms by which it acts in patients with NAFLD have yet to be defined (55).

- N-acetylcysteine increases glutathione levels in the liver protecting it from oxidative stress. A dose of 1-1.2 g/day decrease ALT and GGT levels, although these positive effects appear not to be durable and effective in reducing histologic inflammation (37).

- Betaine is a metabolite of choline which protects the liver from triglyceride deposition and oxidative stress by increasing the levels of S-adenosylmethionine (36). It has been observed that it produces a decrease in transaminases and FLD, as well as a histological improvement (37).

- Angiotensin II receptor blockers (ARBs): Inhibit the proliferation of stellate cells, reducing inflammation and fibrosis. It has been demonstrated that treatment with losartan significantly reduces plasma concentrations of TGF-b1, serum ferritin and transaminases, as well as necroinflammation and liver fibrosis in patients with NAFLD and hypertension. Telmisartan is an angiotensin receptor blocker type I and a PPAR-c modulator, which has also demonstrated effects of PPAR-a activators on the liver with improvement in cytolysis and necroinflammation, and more effectively compared with losartan (37).

\section{Other therapeutic options in research}

- Antibiotics and probiotics: In animal models, treatment with polymyxin B and metronidazole reduces intestinal bacterial overgrowth, with a decrease in endotoxin and the degree of FLD and liver inflammation, probably due to the lower production of TNF- $\alpha$. High levels of Enterobacteriaceae were identified in more cirrhotic rats than in healthy ones, and treatment with bifidobacteria reduced levels of Enterobacteriaceae (27). Oral preparations of probiotics, non-pathogenic Escherichia coli and gentamicin reducing bacterial translocation in animal models attenuated liver damage by decreasing levels of TNF- $\alpha$, IL-6, IL-10 and IL-12. These data suggest that intestinal bacterial overgrowth and impaired epithelial barrier may be a potential therapeutic target for patients with NAFLD (27).

- Melatonin: Mitochondrial respiratory chain (MRC) activity is decreased in patients with NAFLD, due to the fact that peroxynitrite plays a pathogenic role. Melatonin - a peroxynitrite sensor - can restore liver morphology, mitochondrial dysfunction and MRC activity in animals (52).

- Farnesoid X receptor (FXR) agonists form part of the nuclear hormone receptor family, regulated by bile acids, whose activation plays an important role in cholesterol homeostasis, glucose metabolism and insulin sensitivity. INT-747 -a semi-synthetic FXR agonistincreases insulin secretion, it enhances adipocyte lipid storage and secretion of adiponectin and leptin. INT-747 treatment has been associated with an improvement in glucose levels, of fibrosis markers and weight loss (56).

- Phlebotomy and iron chelation therapy: Iron depletion is beneficial in relation to coronary heart disease, endothelial dysfunction, insulin secretion, insulin action and metabolic control of DM-2. Phlebotomy in patients with NAFLD and hepatic iron overload associated with insulin resistance may decrease the risk of fibrosis, cancer or cardiovascular disease (57). Deferoxamine (iron-chelating agent) attenuates intestinal mucosal damage, reducing bacterial translocation and endotoxin levels of portal and systemic circulation. This observation suggests that iron is involved in the pathogenesis of NAFLD (57).

- Omega-3 supplements: This polyunsaturated fatty acid has been studied in patients with NAFLD, compared with placebo, with the observation that Omega-3 may reduce intrahepatic fat even when the ideal dose of treatment has not been defined (58).

\section{Liver transplantation}

Liver transplantation is indicated in patients with cirrhosis due to NAFLD in terminal stages. Although transplant outcome is usually good, steatosis may recur in the majority of patients, without developing steatohepatitis or fibrosis. The causes of recurrence are unknown although they may be multiple: hypertriglyceridaemia, obesity, diabetes and corticosteroid treatment $(15,36)$.

Severe, and even moderate, steatosis may be caused by primary graft dysfunction, as organ donors with macrovesicular steatosis $>10$ to $30 \%$ are rejected (depending on the centre) or patients with BMI $>28$ (8). Possible mechanisms to explain graft dysfunction may be reduced ability to generate adenosine triphosphate and generation of toxic metabolites. By contrast, livers with microvesicular steatosis may be used more safely for transplantation (59). In transplanted livers due to indications other than NAFLD, the risk of developing steatosis again is $20 \%$, and may be related to the weight gain that occurs during the first 2 years after transplantation, as well as the use of corticosteroids (59).

\section{CONCLUSIONS}

NAFLD is a disease with an increasing prevalence, which may be part of the metabolic syndrome and associated with DM-2 and obesity. Factors such as oxidative stress and pro-inflammatory cytokines may intervene in its pathogenesis. 
The liver biopsy continues to be the gold-standard test for NAFLD. Nevertheless, a growing number of diagnostic "non invasive" tests may help limit the use of the biopsy. Among these we can point out the serologic tests such as Steato Test, Fibro Test, and NFL or imaging tests such as the ultrasound and FibroScan.

The treatment of NAFLD is based on the correction of the causing factors (diabetes, obesity, metabolic syndrome). Modifications in life-style, especially diet and exercise are still the pillars for correcting and preventing the progression of NAFLD into cirrhosis and hepatocelular carcinoma. The different pharmacological approaches that have been studied have not been effective. In advanced stages of hepatic disease secondary to NAFLD, liver transplant is an effective alternative.

\section{REFERENCES}

1. Bellentani S, Saccoccio G, Masutti F, Croce LS, Brandi G, Sasso F, et al. Prevalence of and risk factors for hepatic steatosis in Northern Italy. Ann Intern Med 2000;132:112-7.

2. Bugianesi E, Leone N, Vanni E, Marchesini G, Brunello F, Carucci P, et al. Expanding the natural history of nonalcoholic steatohepatitis: From cryptogenic cirrhosis to hepatocellular carcinoma. Gastroenterology 2002:123:134-40.

3. Bellentani S, Scaglioni F, Marino M, Bedogni G. Epidemiology of non-alcoholic fatty liver disease. Dig Dis 2010;28:155-61.

4. Romero FP. Will non-invasive testing replace liver biopsy in the diagnosis and follow-up of non-alcoholic steatohepatitis (NASH)? Rev Esp Enferm Dig 2009;101:521-7.

5. Moreno-Sanchez D. Epidemiology and natural history of primary nonalcoholic fatty liver disease. Gastroenterol Hepatol 2006;29:244-54

6. Daly AK, Ballestri S, Carulli L, Loria P, Day CP. Genetic determinants of susceptibility and severity in nonalcoholic fatty liver disease. Expert Rev Gastroenterol Hepatol 2011;5:253-63.

7. Solis-Herruzo JA, Solis-Munoz P. Genetic factors in non-alcoholic fatty liver disease. Rev Esp Enferm Dig 2008;100:195-201.

8. Zain SM, Mohamed R, Mahadeva S, Cheah PL, Rampal S, Basu RC, et al. A multi-ethnic study of a PNPLA3 gene variant and its association with disease severity in non-alcoholic fatty liver disease. Hum Genet 2012;131:1145-52.

9. Machado MV, Cortez-Pinto H. Non-invasive diagnosis of non-alcoholic fatty liver disease - A critical appraisal. J Hepatol 2012;Nov 23 (Epub ahead of print).

10. Targher G, Marra F, Marchesini G. Increased risk of cardiovascular disease in non-alcoholic fatty liver disease: causal effect or epiphenomenon? Diabetologia 2008;51:1947-53.

11. Solís Herruzo JA, García Ruiz I, Pérez Carreras M, Munoz Yague MT. Non-alcoholic fatty liver disease. From insulin resistance to mitochondrial dysfunction. Rev Esp Enferm Dig 2006;98:844-74.

12. Hashimoto E, Tokushige K. Prevalence, gender, ethnic variations, and prognosis of NASH. J Gastroenterol 2011;46(Supl. 1):63-9.

13. Schindhelm RK, Dekker JM, Nijpels G, Bouter LM, Stehouwer CD, Heine RJ, et al. Alanine aminotransferase predicts coronary heart disease events: A 10-year follow-up of the Hoorn Study. Atherosclerosis 2007;191:391-6.

14. Bonora E, Targher G. Increased risk of cardiovascular disease and chronic kidney disease in NAFLD. Nat Rev Gastroenterol Hepatol 2012;9:372-81

15. Inoue K, Kodama T, Daida H. Pentraxin 3: A novel biomarker for inflammatory cardiovascular disease. Int J Vasc Med 2012;2012:657025.doi. 10:1155/2012/657025. Epub 2012 Jan 14.

16. Medina J, Fernández-Salazar LI, García-Buey L, Moreno-Otero R. Approach to the pathogenesis and treatment of nonalcoholic steatohepatitis. Diabetes Care 2004;27:2057-66.
17. Day CP, James OF. Steatohepatitis: a tale of two "hits"? Gastroenterology 1998;114:842-5.

18. Medina J, Moreno-Otero R. Pathophysiological basis for antioxidant therapy in chronic liver disease. Drugs 2005;65:2445-61.

19. Majano PL, García-Monzón C, López-Cabrera M, Lara-Pezzi E, Fernández-Ruiz E, Garcia-Iglesias C, et al. Inducible nitric oxide synthase expression in chronic viral hepatitis. Evidence for a virus-induced gene upregulation. J Clin Invest 1998;101:1343-52.

20. García-Monzón C, Majano PL, Zubia I, Sanz P, Apolinario A, Moreno-Otero R. Intrahepatic accumulation of nitrotyrosine in chronic viral hepatitis is associated with histological severity of liver disease. J Hepatol 2000;32:331-8.

21. Carter-Kent C, Zein NN, Feldstein AE. Cytokines in the pathogenesis of fatty liver and disease progression to steatohepatitis: Implications for treatment. Am J Gastroenterol 2008;103:1036-42.

22. Sanz-Cameno P, Medina J, García-Buey L, García-Sánchez A, Borque MJ, Martin-Vilchez S, et al. Enhanced intrahepatic inducible nitric oxide synthase expression and nitrotyrosine accumulation in primary biliary cirrhosis and autoimmune hepatitis. J Hepatol 2002;37:723-9.

23. García-Monzón C, Martin-Pérez E, Iacono OL, Fernández-Bermejo M, Majano PL, Apolinario A, et al. Characterization of pathogenic and prognostic factors of nonalcoholic steatohepatitis associated with obesity. J Hepatol 2000;33:716-24.

24. Kaser S, Moschen A, Cayon A, Kaser A, Crespo J, Pons-Romero F, et al. Adiponectin and its receptors in non-alcoholic steatohepatitis. Gut 2005;54:117-21.

25. Luo R, Li X, Jiang R, Gao X, Lu Z, Hua W. Serum concentrations of resistin and adiponectin and their relationship to insulin resistance in subjects with impaired glucose tolerance. J Int Med Res 2012;40:621-30.

26. Aller R, De Luis DA, Izaola O, Gonzalez Sagrado M, Conde R, Pacheco D, et al. Lys656Asn polymorphism of leptin receptor, leptin levels and insulin resistance in patients with non alcoholic fatty liver disease. Eur Rev Med Pharmacol Sci 2012;16:335-41.

27. Ilan Y. Leaky gut and the liver: A role for bacterial translocation in nonalcoholic steatohepatitis. World J Gastroenterol 2012;18:2609-18.

28. 28. Mallat A, Lotersztajn S. Endocannabinoids and liver disease. I. Endocannabinoids and their receptors in the liver. Am J Physiol Gastrointest Liver Physiol 2008;294:G9-12.

29. Fierbinteanu-Braticevici C, Dina I, Petrisor A, Tribus L, Negreanu L, Carstoiu C. Noninvasive investigations for non alcoholic fatty liver disease and liver fibrosis. World J Gastroenterol 2010;16:4784-91.

30. Festi D, Schiumerini R, Marzi L, Di Biase AR, Mandolesi D, Montrone L, et al. Review article: The diagnosis of non-alcoholic fatty liver disease - availability and accuracy of non-invasive methods. Aliment Pharmacol Ther 2013;37:392-400.

31. Wilson SR, Rosen IE, Chin-Sang HB, Arenson AM. Fatty infiltration of the liver -an imaging challenge. J Can Assoc Radiol 1982;33:227-32.

32. Friedrich-Rust M, Romen D, Vermehren J, Kriener S, Sadet D, Herrmann E, et al. Acoustic radiation force impulse-imaging and transient elastography for non-invasive assessment of liver fibrosis and steatosis in NAFLD. Eur J Radiol 2012;81:e325-31.

33. de Ledinghen V, Vergniol J, Foucher J, Merrouche W, le Bail B. Non-invasive diagnosis of liver steatosis using controlled attenuation parameter (CAP) and transient elastography. Liver Int 2012;32:911-8.

34. Brunt EM, Janney CG, Di Bisceglie AM, Neuschwander-Tetri BA, Bacon BR. Nonalcoholic steatohepatitis: A proposal for grading and staging the histological lesions. Am J Gastroenterol 1999;94:2467-74.

35. Kleiner DE, Brunt EM, Van Natta M, Behling C, Contos MJ, Cummings OW, et al. Design and validation of a histological scoring system for nonalcoholic fatty liver disease. Hepatology 2005;41:1313-21.

36. Cobo Martin M, Fernandez Gil P, Crespo J. Treatment of fatty liver disease. Gastroenterol Hepatol 2008;31:229-38.

37. Kaser S, Ebenbichler CF, Tilg H. Pharmacological and non-pharmacological treatment of non-alcoholic fatty liver disease. Int J Clin Pract 2010;64:968-83

38. Mingrone G, Panunzi S, De Gaetano A, Guidone C, Iaconelli A, Leccesi L, et al. Bariatric surgery versus conventional medical therapy for type 2 diabetes. N Engl J Med 2012;366:1577-85.

39. Carlsson LM, Peltonen M, Ahlin S, Anveden A, Bouchard C, Carlsson $\mathrm{B}$, et al. Bariatric surgery and prevention of type 2 diabetes in Swedish obese subjects. N Engl J Med 2012;367:695-704. 
40. Shyangdan D, Clar C, Ghouri N, Henderson R, Gurung T, Preiss D, et al. Insulin sensitisers in the treatment of non-alcoholic fatty liver disease: a systematic review. Health Technol Assess 2011;15:1-110.

41. Lutchman G, Modi A, Kleiner DE, Promrat K, Heller T, Ghany M, et al. The effects of discontinuing pioglitazone in patients with nonalcoholic steatohepatitis. Hepatology 2007;46:424-9.

42. Ratziu V, Charlotte F, Bernhardt C, Giral P, Halbron M, Lenaour G, et al. Long-term efficacy of rosiglitazone in nonalcoholic steatohepatitis: Results of the fatty liver improvement by rosiglitazone therapy (FLIRT 2) extension trial. Hepatology 2010;51:445-53

43. Akcam M, Boyaci A, Pirgon O, Kaya S, Uysal S, Dundar BN. Therapeutic effect of metformin and vitamin $\mathrm{E}$ versus prescriptive diet in obese adolescents with fatty liver. Int J Vitam Nutr Res 2011;81:398-406.

44. 44. Duseja A, Das A, Dhiman RK, Chawla YK, Thumburu KT, Bhadada $\mathrm{S}$, et al. Metformin is effective in achieving biochemical response in patients with nonalcoholic fatty liver disease (NAFLD) not responding to lifestyle interventions. Ann Hepatol 2007;6:222-6.

45. Neuschwander-Tetri BA, Caldwell SH. Nonalcoholic steatohepatitis: Summary of an AASLD Single Topic Conference. Hepatology 2003;37:1202-19.

46. Harrison SA, Fecht W, Brunt EM, Neuschwander-Tetri BA. Orlistat for overweight subjects with nonalcoholic steatohepatitis: A randomized, prospective trial. Hepatology 2009;49:80-6.

47. Argo CK, Loria P, Caldwell SH, Lonardo A. Statins in liver disease: A molehill, an iceberg, or neither? Hepatology 2008;48:662-9.

48. Nelson A, Torres DM, Morgan AE, Fincke C, Harrison SA. A pilot study using simvastatin in the treatment of nonalcoholic steatohepatitis: A randomized placebo-controlled trial. J Clin Gastroenterol 2009:43:990-4

49. Gómez-Domínguez E, Gisbert JP, Moreno-Monteagudo JA, GarcíaBuey L, Moreno-Otero R. A pilot study of atorvastatin treatment in dyslipemid, non-alcoholic fatty liver patients. Aliment Pharmacol Ther 2006;23:1643-7.
50. Nakahara T, Hyogo H, Kimura Y, Ishitobi T, Arihiro K, Aikata H, et al. Efficacy of rosuvastatin for the treatment of non-alcoholic steatohepatitis with dyslipidemia: An open-label, pilot study. Hepatol Res 2012;42:1065-72.

51. Athyros VG, Tziomalos K, Gossios TD, Griva T, Anagnostis P, Kargiotis $\mathrm{K}$, et al. Safety and efficacy of long-term statin treatment for cardiovascular events in patients with coronary heart disease and abnormal liver tests in the Greek Atorvastatin and Coronary Heart Disease Evaluation (GREACE) Study: A post-hoc analysis. Lancet 2010;376:1916-22.

52. Solís Herruzo JA, Solís Munoz P. Melatonin and oxidative stress. Rev Esp Enferm Dig 2009;101:453-9.

53. Zein CO, Lopez R, Fu X, Kirwan JP, Yerian LM, McCullough AJ, et al. Pentoxifylline decreases oxidized lipid products in nonalcoholic steatohepatitis: New evidence on the potential therapeutic mechanism. Hepatology 2013;56:1291-9.

54. Ratziu V, de Ledinghen V, Oberti F, Mathurin P, Wartelle-Bladou C, Renou C, et al. A randomized controlled trial of high-dose ursodesoxycholic acid for nonalcoholic steatohepatitis. J Hepatol 2011;54:1011-9.

55. Chung MY, Yeung SF, Park HJ, Volek JS, Bruno RS. Dietary alphaand gamma-tocopherol supplementation attenuates lipopolysaccharide-induced oxidative stress and inflammatory-related responses in an obese mouse model of nonalcoholic steatohepatitis. J Nutr Biochem 2010;21:1200-6.

56. Adorini L, Pruzanski M, Shapiro D. Farnesoid X receptor targeting to treat nonalcoholic steatohepatitis. Drug Discov Today 2012;17:988-97.

57. Guillygomarc'h A, Mendler MH, Moirand R, Laine F, Quentin V, David V, et al. Venesection therapy of insulin resistance-associated hepatic iron overload. J Hepatol 2001;35:344-9.

58. Parker HM, Johnson NA, Burdon CA, Cohn JS, O'Connor HT, George J. Omega-3 supplementation and non-alcoholic fatty liver disease: A systematic review and meta-analysis. J Hepatol 2012;56:944-51.

59. Sanyal AJ. AGA technical review on nonalcoholic fatty liver disease. Gastroenterology 2002;123:1705-25. 